\title{
Sélection d'une souche de Bacillus thermophile pour la mesure de l'efficacité stérilisatrice des installations U.H.T.
}

\author{
par \\ R. SOARES DE MELO*, O. CERF \\ et J. HERMIER
}

Institut National de la Recherche Agronomique, Station Centrale de Recherches Laitières - 78350 Jouy-en-Josas (France)

\section{Rés u m é}

Une souche de Bacillus coagulans, atypique pour la température optimale de croissance $\left(65^{\circ} \mathrm{C}\right)$, et pour sa thermorésistance, a été sélectionnée pour servir à la mesure de l'efficacité stérilisatrice des installations U.H.T. Les caractéristiques de la thermorésistance des spores dans le lait U.H.T. sont : $1,9 \leqslant \mathrm{D}_{120} \leqslant 2,5 \mathrm{mn}, 10 \leqslant \mathrm{z} \leqslant 11,5^{\circ} \mathrm{C}$. La croissance dans le lait U.H.T. est accompagnée d'une modification visible et caractéristique du lait. Le nombre de spores survivantes peut être obtenu par numération dans un milieu de culture solide ou dans le lait U.H.T. si on lui a ajouté $5,6 \mathrm{mg} / 1 \mathrm{de}$ fer, $20 \mathrm{mg} / 1 \mathrm{de}$ calcium et $24 \mathrm{mg} / 1$ de magnésium. L'addition de ces cations au lait ne modifie pas la thermorésistance des spores.

\section{S u m m a r y}

SELECTION OF A THERMOPHILIC BACILLUS STRAIN FOR MEASURING THE STERILIZING EFFICIENCY OF U.H.T. PLANTS

A strain of Bacillus coagulans, atypical for the optimum growth temperature $\left(65^{\circ} \mathrm{C}\right)$, and for the heat resistance, was selected for measuring the sterilizing efficiency of U.H.T. plants. The heat resis-

* Boursier de la Junta Nacional de Investigaçào Cientifica e Tecnologica. Adresse actuelle : Instituto Nacional de Investigaçào Industrial, Rua Vale Formoso de Bayxo 1, Lisboa 6 (Portugal). 
tance of spores in U.H.T. milk is characterized by : $1,9 \leqslant \mathrm{D}_{120} \leqslant 2.5 \mathrm{~min}$ and $10 \leqslant z \leqslant 11.5^{\circ} \mathrm{C}$. The growth in U.H.T. milk results in a visible and distinctive modification of the milk. Surviving spores number may be obtained from enumeration by plate count method in a solid medium or by M.P.N. method in U.H.T. milk containing iron $5.6 \mathrm{mg} / 1$, calcium $20 \mathrm{mg} / \mathrm{l}$, and magnesium $24 \mathrm{mg} / \mathrm{l}$. These cations in milk do not modify the heat resistance of spores.

\section{INTRODUCTION}

Le seul moyen de s'assurer qu'une installation a une efficacité stérilisatrice convenable sur le lait consiste à ajouter au lait cru une quantité connue de spores bactériennes thermorésistantes, puis à incuber les bouteilles de produit fini, et enfin à contrôler dans quelle proportion de récipients des spores survivantes se sont développées. On obtient ainsi une mesure expérimentale de l'efficacité stérilisatrice de l'installation (logarithme du rapport concentration de spores dans le lait cru sur concentration des spores survivantes dans le lait traité), ce qui permet d'obtenir une estimation du risque de nonstérilité dans les conditions usuelles d'emploi de l'installation, connaissant par ailleurs la teneur moyenne du lait en spores thermorésistantes (Galesloot, 1956).

Les spores des souches bactériennes à utiliser pour ce type d'essai devraient remplir dans l'idéal les conditions suivantes :

$1^{\circ}$ Avoir un temps de réduction décimale d'environ $0,5 \mathrm{~s}$ à $150^{\circ} \mathrm{C}$, de sorte que dans l'appareil commercial réalisant actuellement la plus forte combinaison temps-température, l'efficacité stérilisatrice sur ces spores serait de 5 environ : le nombre de spores à ajouter au lait cru ne serait donc pas trop élevé eu égard à la difficulté qu'il y a toujours à préparer des spores en grandes quantités.

$2^{\circ}$ Avoir des courbes de survie rectilignes (courbes de type A, fig. 1), pour éviter les difficiles calculs imposés par les autres types de courbes de survie (Burton, 1958).

3. Ne pas avoir leur développement inhibé par le lait.

4. Conserver la propriété précédente lorsqu'elles ont été soumises à un traitement thermique qui lèse les spores survivantes.

$5^{\circ}$ Provoquer dans un délai raisonnable une altération visible et reconnaissable du lait.

Le tableau 1 montre que les caractéristiques indiquées pour les souches utilisées par différents auteurs pour mesurer l'efficacité stérilisatrice de diverses installations U.H.T. ne répondent pas à la totalité des exigences que nous venons de citer. C'est pourquoi nous avons entrepris de chercher une souche qui y satisfasse davantage. 
TABLEAU 1

Propriétés des souches utilisées pour le contrôle de l'efficacité stérilisatrice d'installations U.H.T.

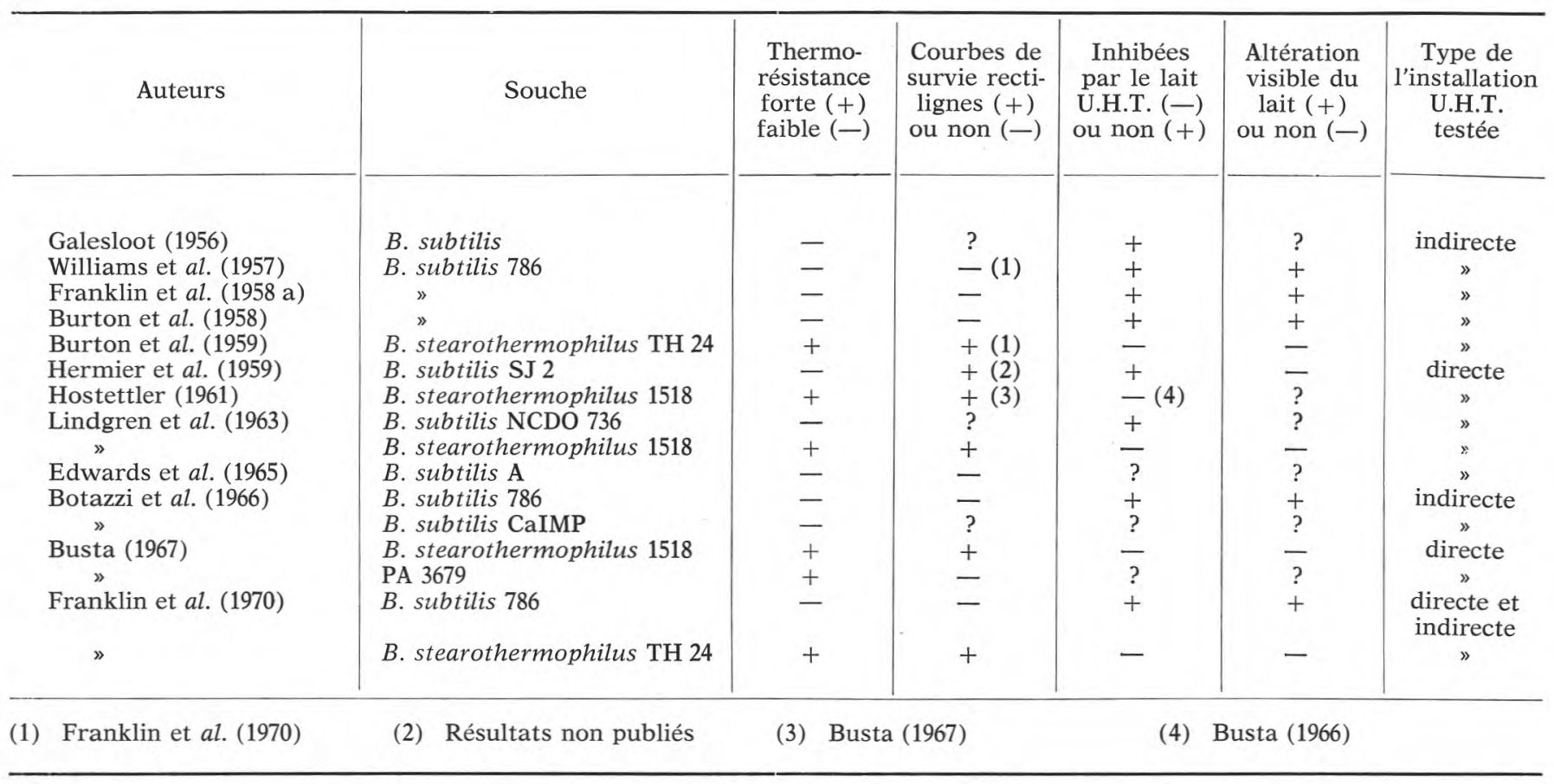




\section{MATERIEL ET METHODES}

\section{1) Souches}

Vingt-cinq souches de Bacillus thermophiles provenant de la collection CNRZ ont été examinées. Les souches 24 et 115 à 118 proviennent de laits U.H.T. insuffisamment chauffés, conditionnés aseptiquement et incubés à $55^{\circ} \mathrm{C}$. Les souches 411 et 416 proviennent de bouteilles de crème chocolatée stérilisée du commerce incubées à $55^{\circ} \mathrm{C}$. Les autres souches proviennent de bouteilles de lait stérilisé du commerce incubées à $55^{\circ} \mathrm{C}$. Onze de ces souches ont été partiellement décrites par Hermier et Bergère (1959) et Hermier (1961).

\section{2) Production des spores}

Les spores des souches 411 à 416 ont été produites sur milieu de composition : Vitamin-free casamino-acids (Difco) $1 \mathrm{~g}$, extrait de levure $5 \mathrm{~g}$, glucose $2,5 \mathrm{~g}, \mathrm{MnSO}_{4} 0,1 \mathrm{~g}, \mathrm{FeSO}_{4} 0,0001 \mathrm{~g}$, gélose $15 \mathrm{~g}$, eau distillée $1000 \mathrm{ml}, \mathrm{pH} \mathrm{6,8}$ (Wang, 1963), celles des autres souches sur milieu GN 6 de composition : extrait de viande Lab Lemco $10 \mathrm{~g}$, gélose $15 \mathrm{~g}$, eau distillée $1000 \mathrm{ml}, \mathrm{pH}$ 6,0 (Hermier, 1961) en boîtes de Roux, à $55^{\circ} \mathrm{C}$. L'inoculum était constitué par $5 \mathrm{ml}$ d'une culture de $16 \mathrm{~h}$ à $55^{\circ} \mathrm{C}$ sur un milieu liquide de même composition que le milieu de sporulation. Pour les souches cultivées sur milieu GN 6, la préparation de l'inoculum était précédée de trois passages sur ce même milieu. La sporulation était suivie par examen au microscope à contraste de phase. Quatorze souches seulement produisaient plus de $10^{7}$ spores par boîtes de Roux. Lorsque la culture n'évoluait plus, les spores étaient récoltées dans de l'eau distillée stérile, chauffées à $80^{\circ} \mathrm{C}$ pendant $10 \mathrm{mn}$, et conservées à $4^{\circ} \mathrm{C}$. En outre les spores de la souches 604 ont été nettoyées par traitement au lysozyme suivi de cinq centrifugations en eau distillée stérile (Cerf, Bergère et Hermier, 1967).

\section{3) Dénombrement des spores}

Un dénombrement des spores a été fait pour chaque souche au moyen d'une cellule de Petroff-Hausser. Pour la numération des spores viables, les techniques suivantes ont été utilisées selon les cas :

- sur boîtes de Petri avec le milieu de Wang (1963) simple ou additionné d'amidon ou de cations comme il sera indiqué avec les résultats à partir de dilutions de spores dans de l'eau distillée stérile. $L^{\prime}$ incubation se fait à $55^{\circ} \mathrm{C}$ pendant $40 \mathrm{~h}$ les boîtes étant enveloppées dans des sachets de polyéthylène pour éviter le dessèchement de la gélose,

- par la méthode des dilutions ou méthode du nombre le plus probable (MPN) avec cinq tubes par dilution. Le milieu de dilution 
et le milieu de numération étaient du lait U.H.T. Pour la détermination de l'aptitude des spores à modifier le lait d'une façon visible, environ $10^{3}$ spores étaient ajoutées à $10 \mathrm{ml}$ de lait. Trois lots de lait U.H.T. de provenances commerciales diverses ont été utilisés. Seule la modification apparente du lait était prise en considération.

\section{4) Mesure de la thermorésistance en ampoules}

Des suspensions de spores de concentration connue étaient faites dans du tampon phosphate $0,02 \mathrm{M}$ à $\mathrm{pH}=7,0$ ou dans du lait U.H.T. fabriqué à la Laiterie Expérimentale de l'I.N.R.A. et réparties dans des ampoules de verre ordinaire à raison de $1,5 \mathrm{ml}$ par ampoule. Le diamètre des ampoules était de $8 \mathrm{~mm}$ et leur longueur de $100 \mathrm{~mm}$. Les ampoules étaient plongées dans un bain thermostaté à $0,1^{\circ} \mathrm{C}$ près, et retirées à des temps préfixés pour être plongées dans de l'eau glacée. Les temps de chauffage étaient corrigés pour tenir compte de la montée en température. Puis les ampoules étaient ouvertes aseptiquement et une partie aliquote de leur contenu était utilisée pour le dénombrement des spores survivantes, par l'une ou l'autre des méthodes décrites dans le paragraphe précédent.

\section{5) Solution de cations}

Dans certains essais le milieu de chauffage ou le milieu de numération étaient complétés par l'addition d'une solution de cations dans des proportions qui seront indiquées avec les résultats. La solution de cations contenait les métaux suivants Fe $100 \mathrm{mM}$, Ca $500 \mathrm{mM}$, $\mathrm{Mg} 1000 \mathrm{mM}$ sous forme de chlorures (Ashton, Busta et Warren, 1968).

\section{6) Identification de la souche $\mathbf{6 0 4}$}

L'identification de la souche 604 a été faite par les techniques de Smith, Gordon et Clark (1952).

\section{RESULTATS}

\section{1) Sélection de la souche 604}

Dans des essais préliminaires, le temps de réduction décimale à $115^{\circ} \mathrm{C}\left(\mathrm{D}_{15}\right)$ en tampon phosphate $0,02 \mathrm{M}$ à $\mathrm{pH}=7,0$ était estimé à partir du nombre de survivants obtenu après un seul temps de chauffage. Ces essais ont conduit à éliminer 16 souches ayant une valeur de $D_{115}$ inférieure à $3 \mathrm{mn}$. Pour les 9 souches retenues, les valeurs de $D_{115}$ étaient comprises entre 3 et $33 \mathrm{mn}$; des essais complémentaires avec un seul temps de chauffage par température ont montré que les valeurs de $\mathrm{z}$ étaient comprises entre 6 et $12^{\circ} \mathrm{C}$. 
TABLEAU 2. - Courbes de survie en tampon phosphate $0,02 \mathrm{M}, \mathrm{pH}=7,0$

\begin{tabular}{|c|c|c|c|c|c|c|c|c|}
\hline Souche & $\begin{array}{c}\text { Température } \\
\left({ }^{\circ} \mathrm{C}\right)\end{array}$ & $\begin{array}{c}\text { Type } \\
\text { de courbe }\end{array}$ & $\underset{(\mathrm{mn})}{\mathrm{D}_{1}}$ & $\begin{array}{c}\mathrm{D}_{2} \\
(\mathrm{mn})\end{array}$ & $\mathbf{N}_{0}$ & $\mathbf{N}_{\mathrm{b}}$ & $\mathbf{N}_{\mathrm{d}}$ & $\begin{array}{c}t_{d} \\
(m n)\end{array}$ \\
\hline $\begin{array}{l}128 \\
171 \\
604 \\
219\end{array}$ & $\begin{array}{l}115 \\
117 \\
117 \\
117\end{array}$ & $\begin{array}{l}\text { A } \\
\text { A } \\
\text { A } \\
\text { A }\end{array}$ & $\begin{array}{l}22 \\
21 \\
11 \\
13\end{array}$ & & $\begin{array}{l}1,1.10^{5} \\
1,4 \cdot 10^{5} \\
1,6.10^{8} \\
1,2.10^{6}\end{array}$ & & & \\
\hline $\begin{array}{l}132 \\
148 \\
154\end{array}$ & $\begin{array}{l}117 \\
117 \\
117\end{array}$ & $\begin{array}{l}\text { B } \\
\text { B } \\
\text { B }\end{array}$ & $\begin{array}{c}8 \\
11,5 \\
6,5\end{array}$ & & $\begin{array}{l}1,1.10^{6} \\
7,2.10^{5} \\
4,5.10^{6}\end{array}$ & $\begin{array}{r}10^{\circ} \\
2,7.10^{7} \\
8.10^{8}\end{array}$ & & \\
\hline $\begin{array}{r}24 \\
139\end{array}$ & $\begin{array}{l}115 \\
115\end{array}$ & $\begin{array}{l}\mathrm{D} \\
\mathrm{D}\end{array}$ & $\begin{array}{r}6 \\
10\end{array}$ & $\begin{array}{l}11,5 \\
15\end{array}$ & $\begin{array}{l}4,4 \cdot 10^{5} \\
1,2 \cdot 10^{8}\end{array}$ & & $\begin{array}{r}8.10^{3} \\
1,3.10^{5}\end{array}$ & $\begin{array}{l}11 \\
10\end{array}$ \\
\hline
\end{tabular}


Les courbes de survie de ces 9 souches après chauffage à $115^{\circ} \mathrm{C}$ ou $117^{\circ} \mathrm{C}$ en tampon phosphate $0,02 \mathrm{M} \mathrm{pH}=7,0$ ont été construites et analysées (tab. 2) conformément aux recommandations de Pflug et Schmidt (1968) qui sont rappelées dans la figure 1 pour les types de courbes rencontrés dans cette étude. Quatre souches avaient une courbe de survie de type A, la meilleure qui convienne pour un organisme-test.

Pour ces 4 souches on a déterminé l'aptitude à la croissance à $55^{\circ} \mathrm{C}$ dans du lait U.H.T. avec altération visible du lait (tab. 3), les

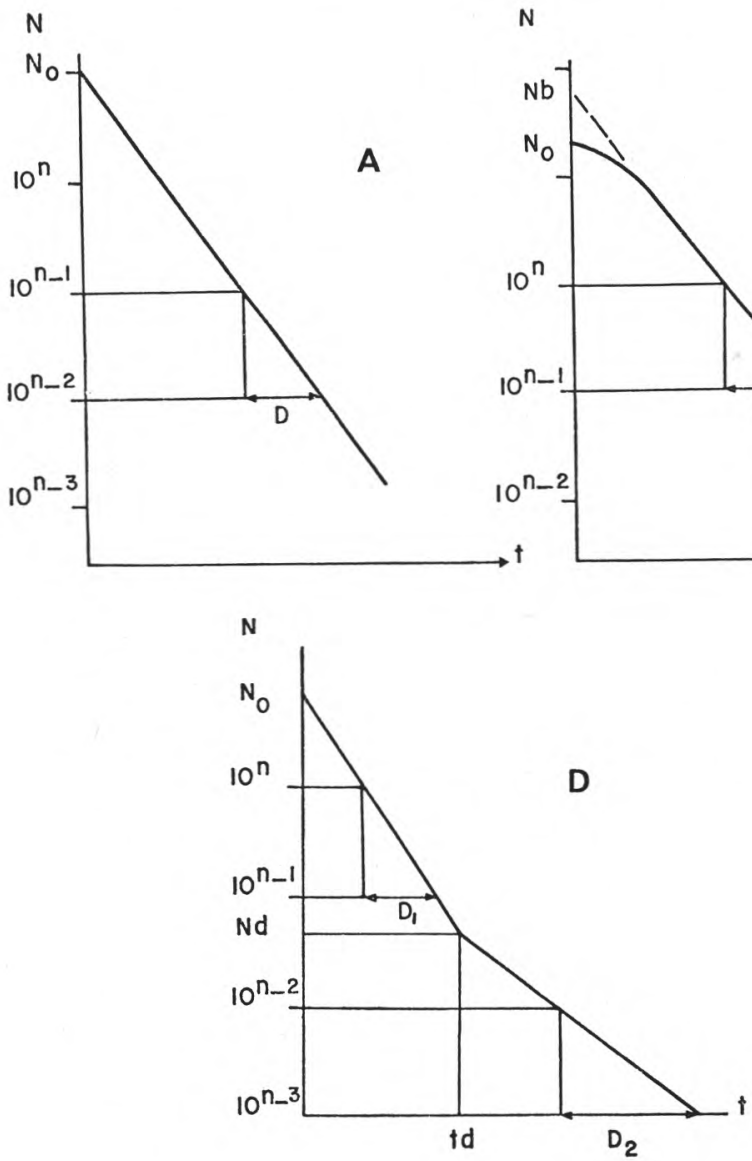

fig. 1

Nomenclature des courbes de survie. Type A : « rectiligne », type B : « à épaulement ", type D : " à traînée ».

$\mathrm{N}$ : nombre de spores par $\mathrm{ml}, \mathrm{t}:$ temps en $\mathrm{mn}$. 
TABLEAU 3. - Aptitude des souches à la croissance dans le lait U.H.T.

\begin{tabular}{l|c|c|c|c}
\hline \multirow{2}{*}{ Souche } & $\begin{array}{c}\text { Modification } \\
\text { visible du } \\
\text { lait }\end{array}$ & \multicolumn{2}{|c}{$\begin{array}{c}\text { Nombre de jours nécessaires à 55 } \\
\text { pour modifier les laits }\end{array}$} \\
\cline { 2 - 4 } 128 & + & lait A & lait B & lait C \\
171 & + & 2 & 8 & 2 \\
604 & + & 2 & - & 6 \\
219 & 3 & 7 & 6 \\
\hline
\end{tabular}

Le signe - indique qu'aucune modification n'est apparue au cours de $21 \mathrm{j}$

TABLEAU 4. - Courbes de survie des spores de la souches 128 en tampon phosphate $0,02 \mathrm{M}, \mathrm{pH}=7,0$

\begin{tabular}{c|c|c|c|c}
\hline $\begin{array}{c}\text { Température } \\
\left({ }^{\circ} \mathrm{C}\right)\end{array}$ & $\begin{array}{c}\text { Type } \\
\text { de courbe }\end{array}$ & $\begin{array}{c}\mathrm{D} \\
(\mathrm{mn})\end{array}$ & $\mathrm{N}_{0}$ & $\mathrm{z}$ \\
\hline 115 & $\mathrm{~A}$ & 22 & $1,1.10^{5}$ & \\
\hline 120 & $\mathrm{~A}$ & 3,5 & $1,1.10^{5}$ & 6,5 \\
125 & $\mathrm{~A}$ & 0,6 & $1,1.10^{5}$ & \\
\hline
\end{tabular}

laits étant ensemencés avec environ $10^{3}$ spores non chauffées. La vitesse de croissance varie avec la souche et l'origine du lait. Les résultats les plus favorables étant obtenus avec les 2 souches 128 et 604 , ces dernières ont été retenues pour une étude plus détaillée (tab. 4 et fig. 2). Les courbes de survie de chacune des 2 souches sont du type A quand elles sont chauffées en tampon phosphate $0,02 \mathrm{M}$ à $\mathrm{pH}=7,0$ à des températures comprises entre $115^{\circ} \mathrm{C}$ et $125^{\circ} \mathrm{C}$. La valeur de $\mathrm{z}$ obtenue à partir des valeurs de $\mathrm{D}$ calculées d'après les courbes de survie est de $6,5^{\circ} \mathrm{C}$ avec la souche 128 et de $8^{\circ} \mathrm{C}$ avec la souche 604 . On a retenu finalement la souche 604 qui, du fait qu'elle a la valeur de $\mathrm{z}$ la plus élevée, est la plus apte aux déterminations de thermorésistance aux températures élevées. 


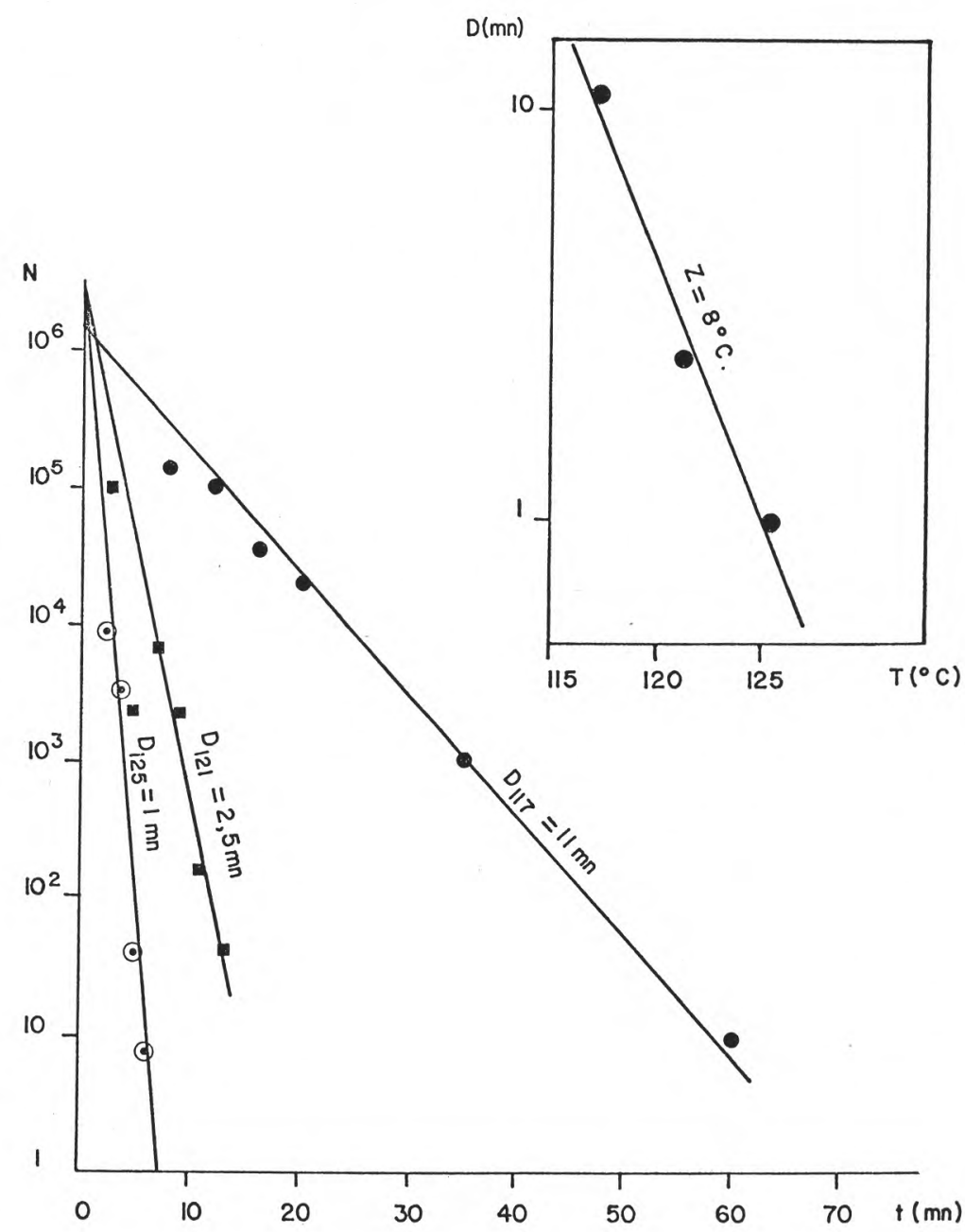

fig. 2

Courbes de survie des spores de la souche 604 en tampon phosphate 0,02 M, $\mathrm{pH}=7,0$. Encadré : courbe de TDT correspondante

\section{2) Thermorésistance de la souche 604 dans le lait}

Les courbes de survie de la souche 604 après chauffage dans du lait U.H.T. ont été établies (fig. 3). Contrairement à ce qui avait été observé dans le cas du chauffage en tampon phosphate, les courbes de survie sont de deux types : $\mathrm{B}$, pour les températures de $117^{\circ} \mathrm{C}$ et $121^{\circ} \mathrm{C}, \mathrm{A}$, pour les températures de $123^{\circ} \mathrm{C}$ et $125^{\circ} \mathrm{C}$. La valeur 


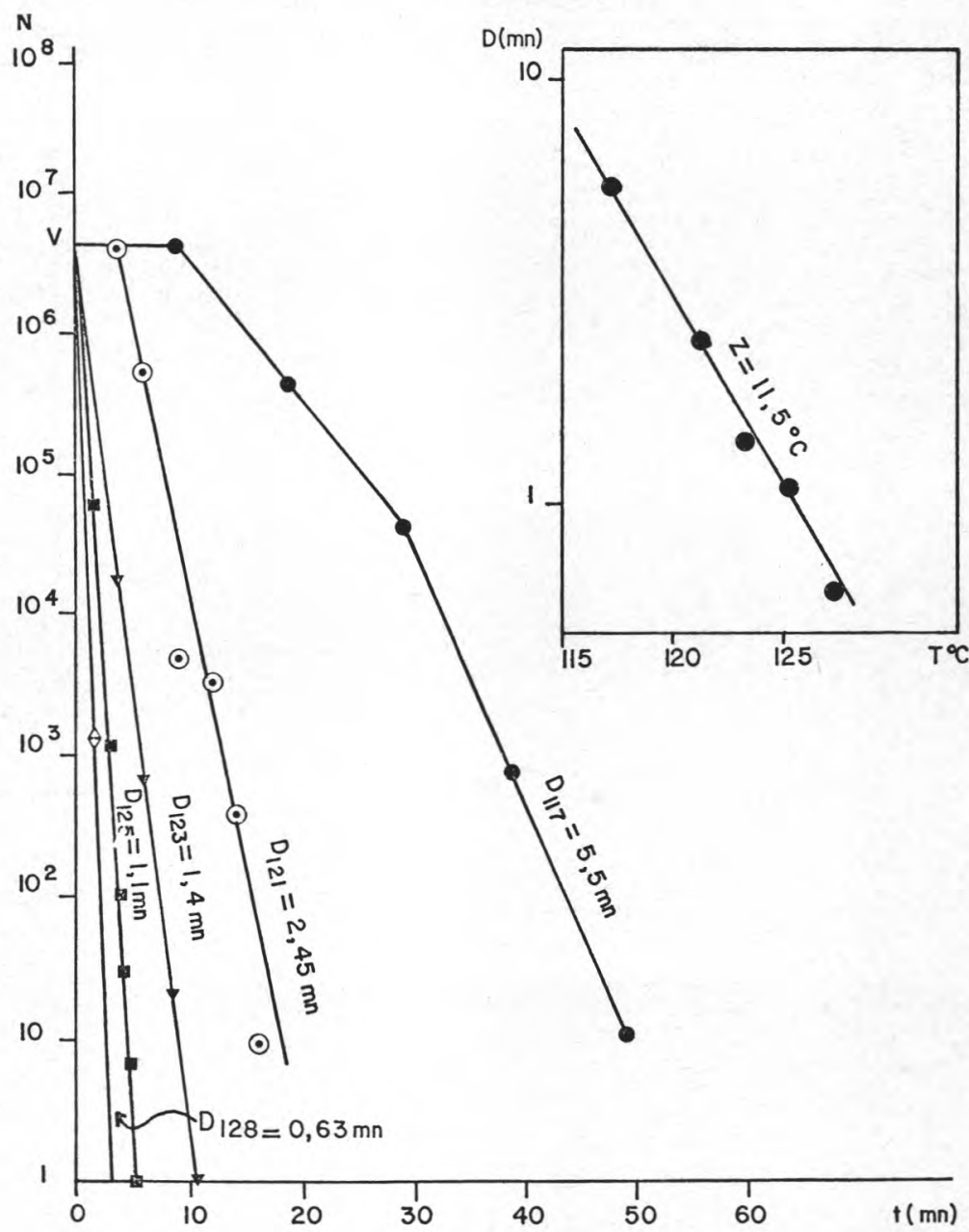

fig. 3

Courbes de survie des spores de la souche 604 en lait U.H.T.

Encadré : courbe de TDT correspondante

de $\mathrm{z}=11,5^{\circ} \mathrm{C}$ est plus élevée que celle obtenue avec un chauffage en tampon $\left(8^{\circ} \mathrm{C}\right)$. Les propriétés de thermorésistance sont donc fortement influencées par le milieu de chauffage. Si le chauffage a lieu en lait autoclavé, la valeur de $\mathrm{z}$ est de $6,5^{\circ} \mathrm{C}$ avec une valeur de $\mathrm{D}$ à $120^{\circ} \mathrm{C}$ de $1,8 \mathrm{mn}$. La thermorésistance est aussi fonction du lot de spores. Pour les chauffages en lait U.H.T. les valeurs extrêmes suivantes ont été obtenues : pour $\mathrm{D}_{120}$ de 1,9 à $2,5 \mathrm{mn}$, pour z de $10^{\circ} \mathrm{C}$ 
à $11,5^{\circ} \mathrm{C}$. Si l'on extrapole à $150^{\circ} \mathrm{C}$ les courbes où est porté logarithme de $\mathrm{D}$ en fonction de la température (courbes de T.D.T.) correspondant à ces valeurs extrêmes, on trouve que $D_{1 \approx}$ est compris entre $0,11 \mathrm{~s}$ et $0.45 \mathrm{~s}$. A titre de comparaison, la valeur de $\mathrm{D}_{150}$ de la souche TH 24 utilisée par Burton et al. (1959) est de 0,14 s (valeur calculée d'après les données publiées).

\section{3) Inhibition des spores de la souche 604 par le lait U.H.T.}

La présence dans le milieu de numération, à raison de $1 \mathrm{ml}$ par boîte de Petri, de lait U.H.T. soumis au chauffage dans les ampoules n'avait aucun effet sur le nombre de colonies obtenues à partir des spores chauffées dans ce lait. En revanche, l'addition au milieu de numération de lait ayant subi uniquement le traitement U.H.T., à raison de $1 \mathrm{ml}$ par boîte de Petri, empêche la formation de colonies à partir de spores chauffées. D'autre part, on n'observe pas de croissance dans du lait U.H.T. ensemencé avec des spores chauffées alors qu'elle est possible dans le cas de spores non chauffées (tab. 3). L'inhibition de la croissance des Bacillus thermophiles par le lait U.H.T. est un phénomène bien connu, mais la souche 604 présente la particularité de ne montrer cette inhibition complète que dans le cas des spores lésées par un traitement thermique. Pour lever cette inhibition, plusieurs solutions ont été envisagées.

\section{a) Numération dans le milieu de Wang contenant du lait U.H.T.}

L'addition d'amidon soluble a été recommandée pour le dénombrement des spores chauffées. L'addition de 0,1 p. 100 d'amidon soluble permet le dénombrement des spores chauffées de la souche 604 , et permet de retrouver le temps de réduction décimale avec une bonne précision (tab. 5).

Il a été montré que l'inhibition de $B$. stearothermophilus par le lait U.H.T. peut être levée par l'addition de cations (Ashton et al., 1968). En fait, l'addition de cations $\left(\mathrm{Fe}^{2+}, \mathrm{Ca}^{2+}, \mathrm{Mg}^{2+}\right)$ au milieu de Wang sans lait U.H.T. inhibe la formation de colonies. Cette inhibition a pu être partiellement levée, conformément aux indications de Ashton et al. (1968), en ramenant le pH du milieu de 6,8 à 6,0. Dans ces conditions le milieu de Wang convient également pour la numération en présence de spores chauffées, sans qu'il y ait modification importante du temps de réduction décimale (tab. 5).

Le résultat est le même si, au lieu d'être ajoutée au milieu de numération, la solution de cations est ajoutée au lait U.H.T. servant de milieu de chauffage à raison de $1 \mathrm{ml}$ pour $1000 \mathrm{ml}$ (tab. 5), et en outre la valeur de $\mathrm{z}$ reste la même que pour les chauffages dans du lait U.H.T. non additionné de cations. Cette concentration correspond à la quantité maximale que l'on peut ajouter au lait sans que le chauffage ne le fasse coaguler. 


\section{TABLEAU 5}

Influence du milieu de chauffage et du milieu de numération sur la valeur des temps de réduction décimale à $120^{\circ} \mathrm{C}$ des spores de la souche 604 .

Les résultats sont exprimés en pourcentage de celui qui est obtenu dans les conditions les plus favorables

\begin{tabular}{|c|c|c|}
\hline \multirow[b]{2}{*}{ Milieu de numération } & \multicolumn{2}{|c|}{ Milieu de chauffage } \\
\hline & lait U.H.T. & $\begin{array}{l}\text { lait U.H.T. } \\
+0,1 \text { p. } 100 \\
\text { cations }\end{array}$ \\
\hline Wang & 100 & 100 \\
\hline Wang +7 p. 100 lait U.H.T. & $<10$ & 95 \\
\hline Wang $+0,1 \mathrm{p} .100$ amidon & 85 & \\
\hline Wang $+0,1$ p. 100 amidon +7 p. 100 lait U.H.T. & 96 & \\
\hline Wang +1 p. 100 cations, $\mathrm{pH}=6,8$ & $<10$ & \\
\hline Wang +1 p. 100 cations +7 p. 100 lait U.H.T., pH $=6,8$ & $<10$ & \\
\hline Wang +1 p. 100 cations, $\mathrm{pH}=6,0$ & 100 & \\
\hline $\begin{array}{l}\text { Wang }+1 \text { p. } 100 \text { cations }+7 \text { p. } 100 \text { lait U.H.T., } p H=6,0 \\
\text { Composition identique à celle du milieu de chauffage }\end{array}$ & $\begin{array}{r}85 \\
10\end{array}$ & \\
\hline Composition identique à celle du milieu de chauffage & $<10$ & 100 \\
\hline
\end{tabular}

\section{b) Numération dans le lait U.H.T.}

La dernière expérience montrant que les cations levaient l'inhibition quand ils étaient ajoutés au milieu de chauffage, la possibilité de dénombrer les survivants dans le lait U.H.T. lui-même a été examinée. On a ainsi constaté que la valeur du temps de réduction décimale obtenue par dénombrement dans le lait U.H.T. additionné de 0,1 p. 100 de la solution de cations en utilisant la méthode des dilutions (MPN), était identique à celle obtenue sur milieu de Wang (tab. 5).

Le tableau 5 indique que les valeurs des temps de réduction décimale sont de même ordre de grandeur lorsque l'inhibition par le lait U.H.T. est levée par une modification soit du milieu de numération, soit du milieu de chauffage. Cela signifie que les courbes de survie sont parallèles entre elles. Mais en fait les courbes de survie ne sont pas superposées : la levée de l'inhibition n'a jamais été complète. La figure 4 montre par exemple les courbes de survie qui correspondent à la modification du milieu de chauffage par addition de cations au lait. On voit que la courbe qui représente le cas où l'inhibition reste la plus forte, c'est-à-dire le chauffage et la numération dans le lait U.H.T. supplémenté, est de type A : elle est donc précisément du type qui convient le mieux pour calculer simplement une efficacité stérilisatrice. Il faut noter aussi que les résultats qui ont servi à établir cette courbe correspondent à une incubation 


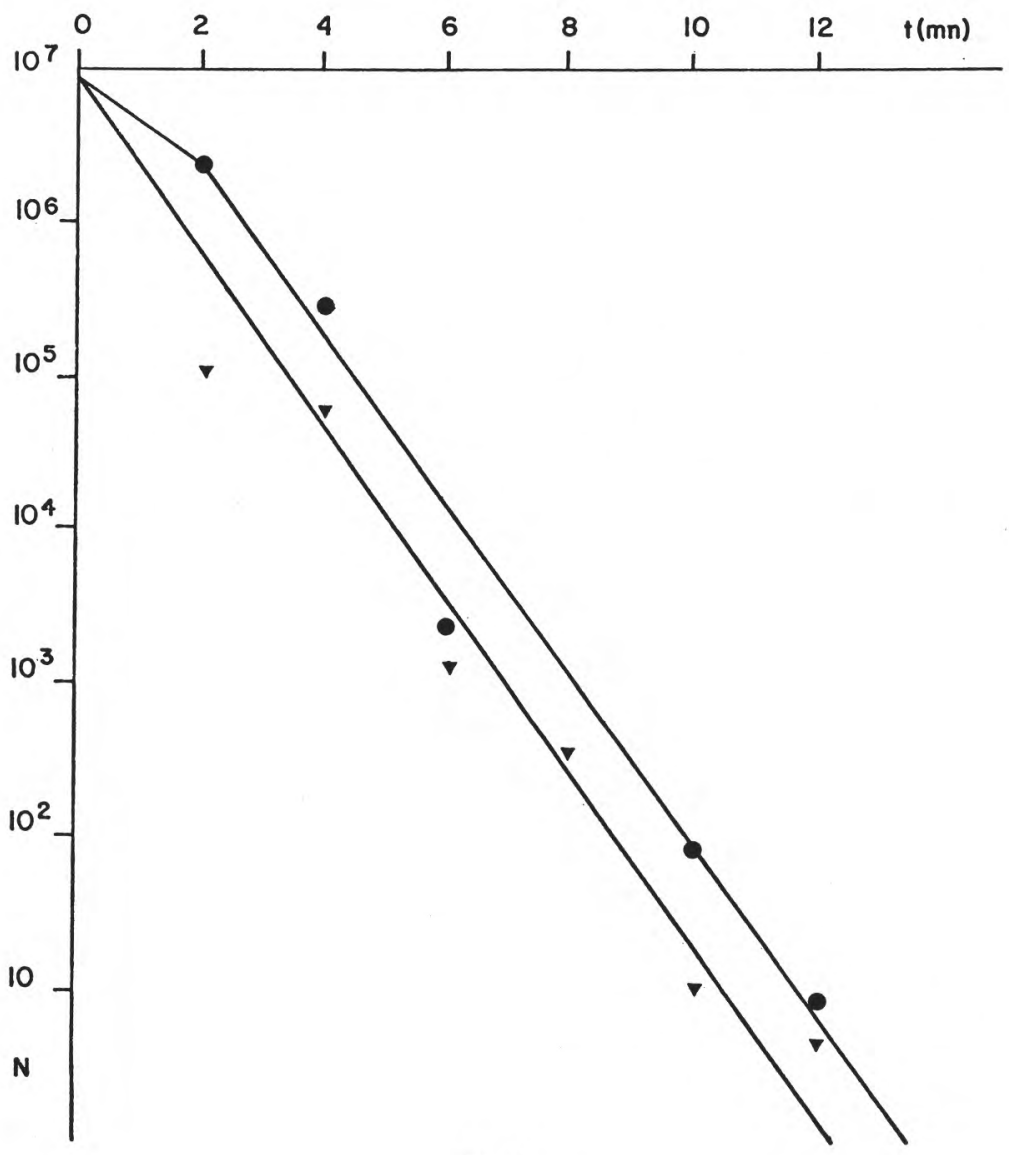

fig. 4

Courbes de survie des spores de la souche 604 en lait U.H.T. additionné de cations. (•) dénombrement sur milieu de Wang (1963) contenant $1 \mathrm{ml}$ de lait U.H.T. non additionné de cations par boîte de Petri ; $(\nabla)$ dénombrement par la méthode des dilutions (MPN) sur lait U.H.T. additionné de cations.

des tubes pendant 1 semaine et qu'une incubation plus longue n'a pas modifié les résultats.

\section{4) Identification de la souche 604}

La souche 604 a tous les caractères culturaux de B. coagulans à deux exceptions près :

- elle est capable de se développer entre $30^{\circ} \mathrm{C}$ et $70^{\circ} \mathrm{C}$ et son optimum de température se situe à $65^{\circ} \mathrm{C}$, 
- elle a les caractères morphologiques du groupe II de la classification de Smith et al. (1952).

Or l'aptitude à se développer à $65^{\circ} \mathrm{C}$ alliée à l'appartenance au groupe II caractérisent $B$. stearothermophilus, si l'on s'en tient à la clef de détermination donnée par Smith et al. (1952). Cependant, la souche 604 produit de l'acétyl-méthyl-carbinol : or l'absence de cette production est d'après Fields et Harris (1972) le caractère le plus important pour la détermination de B. stearothermophilus. Un autre caractère qui empêche de placer la souche 604 dans cette espèce est son aptitude à se développer à $30^{\circ} \mathrm{C}$.

En ce qui concerne les caractères morphologiques, Smith et al. (1952) indiquent qu'ils ont placé arbitrairement $B$. coagulans dans le groupe I bien que de nombreuses souches de cette espèce aient une morphologie les rattachant au groupe II. Ce serait le cas de la souche 604. En ce qui concerne la température de croissance il faut remarquer que :

- Smith et al. (1952) admettent que ce caractère n'est pas à lui seul d'une valeur taxinomique suffisante pour déterminer une espèce,

- de nombreuses souches capables de se développer à $65^{\circ} \mathrm{C}$ n'ont pas les caractères culturaux de B. stearothermophilus (Allen, 1953 ; Galesloot et Labots, 1959 ; Wolf et Barker, 1968 ; Epstein et Grossowicz, 1969 ; Fields, 1970 ; Klaushofer et Hollaus, 1970 ; Harris et Fields, 1972).

On peut donc légitimement classer la souche 604 dans l'espèce B. coagulans, cette souche étant atypique quant à sa température de croissance.

\section{DISCUSSION}

A partir d'un nombre aussi restreint de souches il est remarquable qu'on ait pu en trouver une qui correspondait à l'ensemble des critères fixés. Mais il est également intéressant de constater que 2 des 9 souches dont les courbes de survie ont été établies pour des chauffages en tampon phosphate avaient une cinétique de destruction par la chaleur correspondant au type D (courbes ayant une " trấnée »). C'est là une proportion non négligeable. En outre, les courbes dont les spores ont une courbe de survie rectiligne lors des chauffages en tampon phosphate peuvent avoir des courbes de survie d'un autre type lors des chauffages dans le lait : c'est notamment le cas de la souche 171 dont les spores ont une courbe de survie concave vers le haut pour les chauffages dans le lait (résultat non présenté).

L'inhibition de la croissance des spores des Bacillus thermophiles dans le lait U.H.T. semble être un phénomène assez général (Franklin, 
1970) confirmé dans le cas de la souche 604. Cependant avec cette dernière l'inhibition n'est nette que pour les spores chauffées. Il a été possible de lever cette inhibition soit par l'addition de cations, soit par l'addition d'amidon soluble ; le rapprochement de ces observations inciterait à penser que l'amidon joue ici un rôle dans la levée de l'inhibition par les cations qu'il peut apporter ; l'explication proposée habituellement est que l'amidon se combine avec des acides gras susceptibles d'inhiber le développement des spores.

Put et Wybinga (1963) ont décrit une souche de B. coagulans plus thermorésistance que les souches de la même espèce décrites avant eux. Cette souche était caractérisée par un $\mathrm{D}_{110}$ de $2,4 \mathrm{mn}$ et un $\mathrm{z}$ de $10,5^{\circ} \mathrm{C}$ pour des chauffages dans du tampon phosphate 0,025 $\mathrm{M}$ à $\mathrm{pH}=7,0$. Mais il s'agissait d'une souche mésophile, et l'on admet généralement que les souches mésophiles sont moins thermorésistantes que les souches thermophiles.

Le caractère thermophile de la souche 604 , qui par ailleurs correspond bien à la description de $B$. coagulans, conduit une fois de plus à s'interroger sur le bien-fondé du caractère « croissance à $65^{\circ} \mathrm{C} \gg$ pour la description des espèces bactériennes. Nous suggérons de lui retirer la prééminence qui lui avait été donnée.

Du point de vue technologique, la souche 604 présente un grand intérêt : elle convient tout à fait à l'analyse des performances des appareillages U.H.T. dans les conditions réelles de leur utilisation. Elle répond complètement aux cinq conditions qui ont été fixées dans l'introduction. Ses spores peuvent être dénombrées après chauffage sur un milieu nutritif simple tel le milieu de Wang (1963) additionné soit d'amidon, soit de cations et réglé à $\mathrm{pH}=6,0$. Plus simplement, leur présence peut être détectée par culture dans du lait incubé à $55^{\circ} \mathrm{C}$ et examen visuel du lait. Leur numération peut également être réalisée en utilisant comme milieu de culture le lait même dans lequel elles ont été chauffées en employant la méthode des dilutions (MPN). Dans ces deux derniers cas, il suffira d'avoir ajouté des cations au lait avant son chauffage ; cela ne présente aucun inconvénient puisque non seulement les valeurs du temps de réduction décimale ne sont pas modifiées, mais encore les courbes de survie sont rectilignes sans épaulement.

\section{Remerciements}

Nous remercions Mlle S. Tringidou qui a participé à ce travail en tant que stagiaire et $M$. F. Métro pour sa collaboration technique appréciée. 


\section{Bibliographie}

Allen (M. B.) (1953). - The thermophilic aerobic spore forming bacteria. Bacteriol. Rev., 17, 125-173.

Ashton (D. H.), Busta (F. F.) and WARREN (J. A.) (1968). - Relief of casein inhibition of Bacillus stearothermophilus by iron, calcium and magnesium. Appl. Microbiol., 16, 628-635.

Bottazzi (V.), Dellaglio (F.), Corradini (C.) (1966). - Ricerche sul latte sterilizzato. $1^{\circ}$ Efficienza di sterilizzazione di un impianto U.H.T. Steriplak a scambio indiretto di calore. Sci Tec. Latt.-Cas., 17, 133-142.

BURTON (H.) (1958). - An analysis of the performance of an ultra-high-temperature milk sterilizing plant. II. Calculations of the bactericidal effectiveness. J. Dairy Res., 25, 324337.

Burton (H.), Franklin (J.G.), Williams (D. J.), Chapman (H. R.), Harrison (A. J. W.) and CLEGG (L. F. L.) (1958). An analysis of the performance of an ultra-high-temperature milk sterilizing plant. III. Comparison of experimental and calculated sporicidal effects for a strain of Bacillus subtilis. J. Dairy Res., $25,338-343$.

Burton (H.), Franklin (J. G.), Williams (D. J.), Chapman (H. R.), Harrison (A. J. W.) and CLEGG (L. F. L.) (1959). - An analysis of the performance of an ultra-high-temperature milk sterilizing plant. IV. Comparison of experimental and calculated sporicidal effects for a strain of Bacillus stearothermophilus. J. Dairy Res., 26, 221-226.

Busta (F. F.) (1966). - Milk component(s) inhibitory to Bacillus stearothermophilus. J. Dairy Sci., 49, 751-756.

Busta (F. F.) (1967). - Thermal inactivation characteristics of bacterial spores at ultrahigh temperatures. Appl. Microbiol., 15, 640-645.

Cerf (O.), Bergere (J. L.) et Hermier (J.) (1967). - Thermorésistance des spores de Clostridium tyrobutyricum et Clostridium butyricum. J. Dairy Res., 34, 221-229.

EDWARdS (J. L.) Jr., Busta (F. F.) and SPECK (M. L.) (1965). - Thermal inactivation characteristics of Bacillus subtilis spores at ultrahigh temperatures. Appl. Microbiol., 13, 851-857.

Epstein (I.) and Grossowicz (N.) (1969). - Prototrophic thermophilic Bacillus : isolation, properties, and kinetics of growth. J. Bacteriol., 99, 414-417.

FIELdS (M. L.) (1970). - The flat sour bacteria. Adv. Food Res, 18, 163-217.

FieldS (M. L.) and HARRIS (O.) (1972). - Identification of named cultures of Bacillus calidolactis as Bacillus stearothermophilus. J. Bacteriol., 110, 772-774.

FranKLIN (J.G.) (1970). - Spores in milk : problems associated with U.H.T. processing. J. appl. Bacteriol., 33, 180-191.

Franklin (J. G.), Willtams (D. J.), Chapman (H. R.) and Clegg (L. F. L.) (1958 a). Methods of assessing the sporicidal efficiency of an ultra-high-temperature milk sterilizing plant. II. Experiments with suspensions of spores in milk. J. appl. Bacteriol., 21, 47-50.

Franklin (J. G.), Williams (D. J.) and Clegg (L. F. L.) (1958 b). - Methods of assessing the sporicidal efficiency of an ultra-high-temperature milk sterilizing plant. III. Laboratory determinations of the heat resistance of spores of Bacillus subtilis in water and in milk. J, appl. Bacteriol., 21, 51-57.

Franklin (J. G.), Underwood (H. M.), Perkin (A. G.) and Burton (H.) (1970). Comparison of milk processed by the direct and indirect methods of ultrahigh-temperature sterilization. II. The sporicidal efficiency of an experimental plant for direct and indirect processing. J. Dairy Res., 37, 219-226. 
GaleSLOOT (T. E. A.) (1956). - A simple method of estimating the bacteriological effect of sterilizing processes for milk, applied to the sterilization of milk in ultrahigh temperature - short time (U.H.T.S.T.) - and " in bottle " sterilizers. Neth. Milk Dairy J., 10, 79-100.

GaLESLOOT (T. E.) and Labots (H.) (1959). - Thermophilic bacilli in milk with special reference to the making of sterilized milk and chocolate milk. Neth. Milk Dairy J., 13, 174-179.

HARRIS (O.) and FielDS (M. L.) (1972). - A study of thermophilic aerobic spore forming bacteria isolated from soil and water. Can. J. Microbiol., 18, 917-923.

Hermier (J.) (1961). - L'origine des spores de thermophiles dans le lait stérilisé en bouteilles. Le Lait, 41, 129-144.

Hermier (J.) et Bergere (J. L.) (1959). - Origine des spores de thermophiles et conditions de leur destruction dans la préparation du lait "stérilisé " commercial. $15^{\mathrm{e}}$ Cong. Internat. Lait., 1, 435-441.

Hermier (J.), Verge (J.) et Grosclaude (G.) (1959) - Détermination de la durée de chauffage dans un appareil de stérilisation du lait à très haute température. Le Lait, 39, 20-32.

Hostettler (H.) (1961). - Remplissage aseptique du lait upérisé en emballages Tetra Pak. Laitier Romand J. suisse Lait, 38, (46) 1-12.

Klaushofer (H.) und Hollaus (F.) (1970). - Zur Taxonomie des hochthermophilen, in Zuckerfabrikssäften vorkommenden aeroben Sporenbildner. Z. Zuckerindustrie, 20, 465-470.

Lindgren (B.) and SWARTLing (P.) (1963). - The sterilizing efficiency of the Alfa-Laval Vacu-Therm Instant Sterilizer. Milk Dairy Res. Rep., ${ }^{\circ}$ 69, Alnarp.

Pflug (I. J.) and Schmidt (C. F.) (1968). - Thermal destruction of microorganisms, in LAWRENCE (C. A.) and Block (S.S.). Disinfection, sterilization, and preservation, Lea and Febiger (Philadelphie), 63-105.

Put (H. M. C.) and Wybinga (S.J.) (1963). The occurence of Bacillus coagulans with high heat resistance. J. appl. Bacteriol., 26, 428-434.

Smith (N.R.), GoRdon (R. E.) and ClaRK (F.E.) (1952). - Aerobic sporeforming bacteria. Agriculture Monograph, $\mathrm{n}^{\circ}$ 16, United States Department of Agriculture, U.S. Government Printing Office, Washington.

WANG (D. I. C.) (1963), - The kinetics of death of bacterial spores at elevated temperatures. Ph. D. Thesis, Univ. Pennsylvanie.

Williams (D. J.), Franklin (J. G.), Chapman (H. R.) and Clegg (L. F. L.) (1957), Methods of assessing the sporicidal efficiency of an ultra-high-temperature milk sterilizing plant. I. Experiments with suspensions of spores in water. J. appl. Bacteriol., 20, 43-49.

Wolf (J.) and BARKER (A. N.) (1968). - The genus Bacillus, aids to the identification of its species, in GibBS (B. M.) and SHAPTON (D.A.). Identification metheds for microbiologists, 2 B, Academic Press, Londres et New-York, 95-109. 